\title{
Experimental Path Loss Models for In-Body Communications within 2.36-2.5 GHz
}

\author{
Raúl Chávez-Santiago, Member, IEEE, Concepcion Garcia-Pardo, Alejandro Fornes-Leal, Ana Vallés- \\ Lluch, Günter Vermeeren, Wout Joseph, Senior Member, IEEE, Ilangko Balasingham, Senior \\ Member, IEEE, and Narcís Cardona, Senior Member, IEEE
}

\begin{abstract}
Biomedical implantable sensors transmitting a variety of physiological signals have been proven very useful in the management of chronic diseases. Currently, the vast majority of these in-body wireless sensors communicate in frequencies below $1 \mathrm{GHz}$. Although the radio propagation losses through biological tissues may be lower in such frequencies, e.g., the medical implant communication services (MICS) band of 402-405 MHz, the maximal channel bandwidths allowed therein constrain the implantable devices to low data rate transmissions. Novel and more sophisticated wireless in-body sensors and actuators may require higher data rate communication interfaces. Therefore, the radio spectrum above $1 \mathrm{GHz}$ for the use of wearable medical sensing applications should be considered for in-body applications too. Wider channel bandwidths and smaller antenna sizes may be obtained in frequency bands above $1 \mathrm{GHz}$ at the expense of larger propagation losses. Therefore, in this paper we present a phantom-based radio propagation study for the frequency bands of 2360-2400 $\mathrm{MHz}$, which has been set aside for wearable body area network (BAN) nodes, and the industrial, scientific, medical (ISM) band of 2400-2483.5 MHz. Three different channel scenarios were considered for the propagation measurements: in-body to in-body (IB2IB), in-body to on-body (IB2OB), and in-body to off-body (IB2OFF). We provide for the first time path loss formulas for all these cases.
\end{abstract}

Index Terms-body area network, implantable, in-body, path loss, propagation

\section{INTRODUCTION}

$\mathrm{W}$ IRELESS technologies have revolutionized many aspects of modern life. Medicine and healthcare are not

This work was sponsored by the European COST Action IC1004 (http://www.ic1004.org/) through the short-term scientific mission no. ECOST-STSM-IC1004-17489. R. Chávez-Santiago and I. Balasingham acknowledge financial support from the Research Council of Norway provided to the MELODY Project-Phase II (Contract no. 225885).

R. Chávez-Santiago and I. Balasingham are with the Intervention Centre, Oslo University Hospital, NO-0027 Oslo, Norway (e-mail: raul.chavezsantiago@ rr-research.no; ilangko.balasingham@medisin.uio.no). They are also with the Institute of Clinical Medicine, University of Oslo, and the Norwegian University of Science and Technology (NTNU).

C. Garcia-Pardo, A. Fornes-Leal, and N. Cardona are with iTEAM, Universitat Politècnica de València, 46022 Valencia, Spain (e-mail: cgpardo@iteam.upv.es; alforlea@teleco.upv.es; ncardona@iteam.upv.es).

A. Vallés-Lluch is with the Center for Biomaterials and Tissue Engineering, Universitat Politècnica de València, 46022 Valencia, Spain (email: avalles@ter.upv.es).

G. Vermeeren and W. Joseph are with the Department of Information Technology, Ghent University/iMinds, B-9050 Ghent, Belgium (e-mail: gunter.vermeeren@intec.ugent.be; wout.joseph@intec.ugent.be). the exceptions since multiple applications of radio technologies have had a beneficial impact on the way diagnosis and therapeutic procedures are performed [1]. In the same vein, the use of radio communications combined with biomedical sensors for the continuous monitoring of patients suffering from chronic diseases has gained significant attention as these solutions promise to lower the global expenditure in healthcare [2]-[4]. The interconnection of such wireless biomedical sensors to form a body area network (BAN) has been standardized in IEEE Std 802.15.6 ${ }^{\mathrm{TM}}-2012$ [5], which specifies the physical (PHY) and medium access control (MAC) layers for communication on the surface, inside, or in the peripheral proximity of the human body. According to this standard, wearable BAN nodes can communicate in existing industrial, scientific, medical (ISM) bands as well as other frequency bands approved by national medical and/or regulatory authorities. These include the frequency band of 2360-2400 MHz [6], [7], which the United States Federal Communications Commission (FCC) has set aside for medical wearable sensors in order to complement the already crowded 2400-2483.5 MHz ISM band (simply referred to as the $2.4 \mathrm{GHz}$ ISM band). However, as specified by IEEE Std 802.15.6 $6^{\mathrm{TM}}-2012$, in-body BAN nodes can communicate in the medical implant communication services (MICS) band only, i.e., 402-405 MHz [7]-[10]. Although the expected radio propagation losses through biological tissues in the MICS band may not be as high as within $2360-2400 \mathrm{MHz}$ or $2400-2483.5 \mathrm{MHz}$, the maximal channel bandwidth of 300 $\mathrm{kHz}$ allowed in the MICS band constrains the implantable devices to low data rate transmissions. Therefore, a number of other frequency bands have been considered for communication interfaces with medical implants and in-body electronic pills [11]-[13]. Wider channel bandwidths for novel and more sophisticated in-body wireless sensors and actuators may be exploited in frequency bands above $1 \mathrm{GHz}$ at the expense of larger propagation losses. Despite this drawback, the feasibility of using the radio spectrum above $1 \mathrm{GHz}$ for inbody BAN applications should be investigated because utilizing higher frequencies can also reduce the physical dimensions of implantable antennas that may facilitate the further miniaturization of in-body BAN nodes. For instance, some of the 39 channels of $1 \mathrm{MHz}$ bandwidth available in 2360-2400 MHz could be employed for implant communications besides their intended application as on-body 
radio interfaces; 79 similar channels are available in the 2.4 $\mathrm{GHz}$ ISM band [7]. The idea of using the spectrum around 2.4 $\mathrm{GHz}$ for communication with in-body BAN nodes has been proposed before and a variety of implantable antennas for this purpose have been designed, e.g., [14]-[24]. In addition, propagation losses of body implanted antennas were computed via numerical simulations with single- and three-layer tissue structures for the different ISM bands, namely $433 \mathrm{MHz}, 915$ $\mathrm{MHz}, 2450 \mathrm{MHz}$, and $5800 \mathrm{MHz}$ in [25]. However, no mathematical formulas for the path loss were provided in that study. On the other hand, numerical and experimental path loss investigations with ingested wireless implants in 402 $\mathrm{MHz}, 868 \mathrm{MHz}$, and $2.4 \mathrm{GHz}$ were presented in [26]. A logdistance path loss formula as a function of the propagation distance, $d$, was introduced for the in-body to on-body channel scenario. In this model the path loss exponent, $\gamma$, which indicates the rate at which the path loss, $P L$, increases with $d$, had a value of 2.8 and 2.6 for data obtained from phantom measurements and numerical simulations at $2.4 \mathrm{GHz}$, respectively. Likewise, measurements in a phantom and numerical simulations of path loss for insulated dipole antennas in the ISM band at $2.457 \mathrm{GHz}$ led to path loss formulas for the in-body to in-body channel scenario [27], [28]. In spite of all these research efforts, no comprehensive set of path loss models stemming from measurements or simulations in a single propagation medium for all the possible channel scenarios involving in-body BAN nodes has been reported in the literature. Therefore, to fill this gap we present a radio propagation study for $2360-2500 \mathrm{MHz}$, which covers the two frequency bands discussed above with potential use for implant communications. We performed channel measurements in a liquid phantom, i.e., a chemical solution specially formulated to reproduce the dielectric properties of human muscle tissues [29]. We considered three different channel scenarios: in-body to in-body (IB2IB), in-body to onbody (IB2OB), and in-body to off-body (IB2OFF). We provide for the first time the path loss formulas for all these cases.

The remainder of the paper is organized as follows: In Section II we describe the experimental setup and measurement methodology. Section III presents the path loss formulas for the different in-body channel scenarios. In Section IV we discuss the implications of our findings for the implementation of implant communications in $2.36-2.5 \mathrm{GHz}$. Finally, Section V summarizes our conclusions.

\section{EXPERIMENTAL SETUP AND MEASUREMENT METHODOLOGY}

\section{A. In-Body Channel Scenarios}

The in-body channel scenarios that we considered herein combined the three possible locations of BAN nodes that the Channel Modeling Subgroup of IEEE Std 802.15.6 ${ }^{\mathrm{TM}}-2012$ identified in [30]. Hence, these in-body channel scenarios can be described as:

- IB2IB, a link between two implanted nodes.

- IB2OB, a link between an implanted node and a node in direct contact with the skin or within $2 \mathrm{~cm}$ distance.

- IB2OFF, a link between an implanted node and a node beyond $2 \mathrm{~cm}$ and up to $5 \mathrm{~m}$ from the body surface.

Nevertheless, because of the limitations imposed by the physical size and construction of the phantom, the channel measurements were performed with the best possible approximation to the characteristics of each scenario.

\section{B. Experimental Setup}

The setup used for the IB2IB measurements is shown in Fig. 1, which consisted of a vector network analyzer, a cubeshaped container, phantom aqueous solution, two insulated dipole antennas, and two coaxial cables. For the IB2OB and IB2OFF measurements one of the insulated dipoles was replaced with a free-space coax fed helical antenna with a dielectric support on a finite ground plane and six turns of copper wire. This free-space helical antenna exhibited a reflection coefficient of $\left|S_{11}\right|<-10 \mathrm{~dB}$ within $2.36-2.5 \mathrm{GHz}$ and a gain of $7.7 \mathrm{~dB}$ at $2.4 \mathrm{GHz}$. The physical dimensions of these antennas are shown in Fig. 2. The antennas were connected to an Agilent Technologies ${ }^{\mathrm{TM}}$ ENA E5072A vector network analyzer (VNA), which had a maximal operation frequency of $8.5 \mathrm{GHz}$. Two coaxial cables of 1 meter in length with a maximal operation frequency of $12.4 \mathrm{GHz}$ were used to connect the antennas to the VNA ports 1 and 2, respectively. The cables' frequency response was subtracted from the channel measurements by performing a careful thrucalibration of the VNA. Further details about some components of this measurement setup are given below.

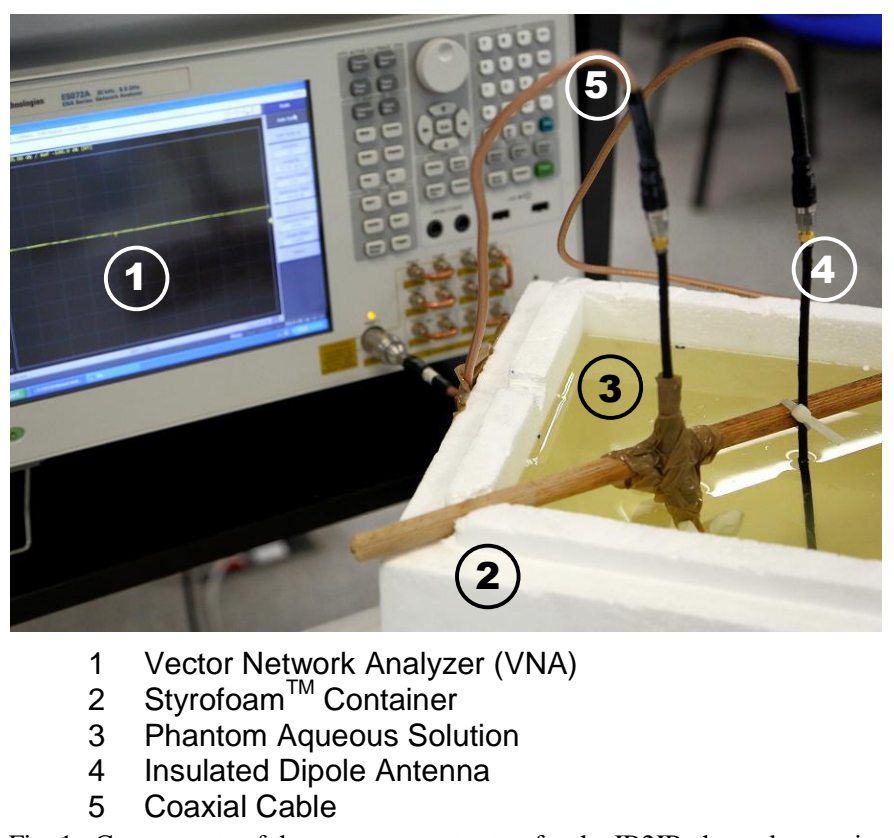

Fig. 1. Components of the measurement setup for the IB2IB channel scenario. Notice that in this case the two implantable antennas were located along an imaginary diagonal line that divided the phantom in two triangular halves. This was done in order to obtain a larger transmission range. However, in the other two cases, i.e., IB2OB and IB2OFF, an implantable antenna and the helical antenna were located along an imaginary line that divided the phantom in two rectangular halves. 


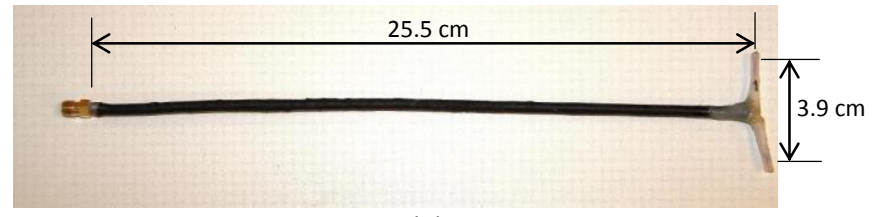

(a)

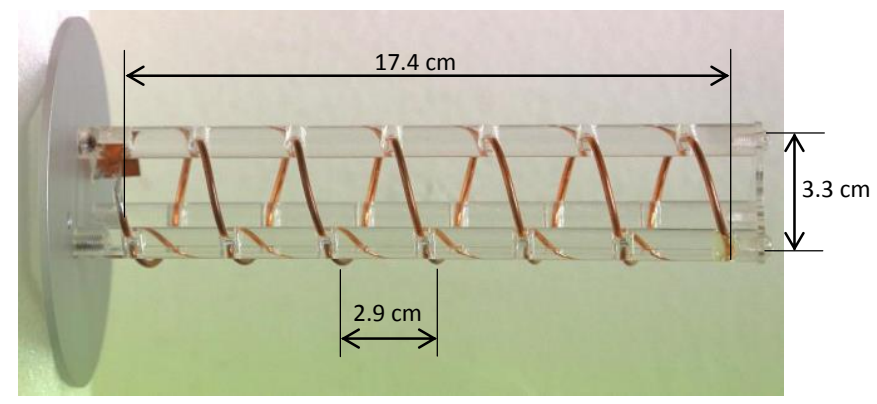

(b)

Fig. 2. (a) Insulated dipole antenna, and (b) free-space coax fed helical antenna.

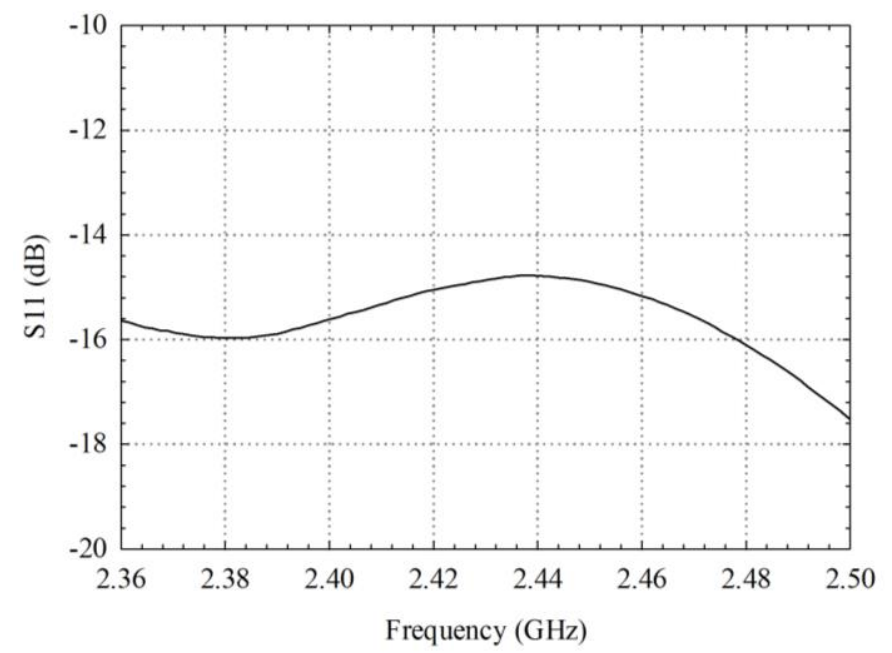

Fig. 3. Measured reflection coefficient of the insulated dipole antenna submerged in a liquid phantom with similar dielectric properties to muscle tissues.

\section{Phantom Aqueous Solution}

In order to approximate the propagation conditions of the human body within $2.36-2.5 \mathrm{GHz}$ an aqueous solution of sucrose $\left(\mathrm{C}_{12} \mathrm{H}_{22} \mathrm{O}_{11}\right)$ and sodium chloride $(\mathrm{NaCl})$ was prepared. Reportedly, a solution with the following weight proportions: $59.5 \%$ of deionized water, $40 \%$ of sucrose, and $0.5 \%$ of sodium chloride, approximates the dielectric characteristics of human muscle tissues at $2.45 \mathrm{GHz}$, i.e., relative permittivity $\varepsilon_{r}=52.7$ and conductivity $\sigma=1.73 \mathrm{~S} / \mathrm{m}$ [24]. However, slight changes in these dielectric parameters may occur because of ambient temperature variations. The prepared solution was poured into a cube-shaped container made of extruded polystyrene foam (Styrofoam ${ }^{\mathrm{TM}}$ ) with wall thickness, $w_{\text {th }}$, of $40 \mathrm{~mm}$. The container's size was $30 \times 30 \times 20$ $\mathrm{cm}^{3}$ (width $\times$ length $\times$ height) and the phantom solution occupied a volume of $22 \times 22 \times 16 \mathrm{~cm}^{3}$. Hereinafter we refer to this arrangement as "the phantom."

\section{Insulated Dipole Antennas}

The insulated dipoles were designed with arms made of perfect electric conductor (PEC) material surrounded by an insulation made of polytetrafluoroethylene $\left(\varepsilon_{r}=2.07\right.$ and $\sigma=0 \mathrm{~S} / \mathrm{m}$ ) [27], [28]; in the physical implementation (Fig. 2(a)), however, the arms were made of copper. These antennas were originally designed to resonate at $2.457 \mathrm{GHz}$ when submerged in a phantom solution with $\varepsilon_{r}=50.8$ and $\sigma=2.01 \mathrm{~S} / \mathrm{m}$. However, through measurements of the reflection coefficient, i.e., $\left|S_{11}\right|$ parameter, we verified that the insulated dipoles covered properly the $2.36-2.5 \mathrm{GHz}$ frequency band when submerged in our liquid phantom described above (Fig. 3).

\section{E. Measurement Methodology}

The measurements consisted of obtaining the forward channel gain in the frequency domain, i.e., $S_{21}(f)$, within the band under analysis. Measurements were performed between $2.36 \mathrm{GHz}$ and $2.5 \mathrm{GHz}$ with $N=20001$ resolution points, thus the frequency resolution was $\Delta f=7 \mathrm{kHz}$. The VNA output power was set to $10 \mathrm{dBm}$ and the noise floor was at $110 \mathrm{dBm}$ within a bandwidth $f_{\mathrm{IF}}=3 \mathrm{kHz}$. All the measured data were recorded for processing and analysis in Matlab ${ }^{\mathrm{TM}}$. Five snapshots of the channel were recorded to improve the signal-to-noise ratio (SNR). The path loss in $\mathrm{dB}$ was then calculated as $P L=-\operatorname{mean}\left\{S_{21}(f)\right\}$. For each channel scenario, a discrete number of path loss points versus the corresponding distance separating the antennas, i.e., $P L\left(d_{i}\right)$, were plotted and a continuous curve was fitted to the resulting scatter plot. The least square fitting method was then used to produce a path loss formula as a function of distance.

\section{PATH LOSS FORMULAS}

\section{A. In-Body to In-Body (IB2IB) Channel Scenario}

In this scenario the two insulated dipoles were submerged in the phantom so that the center of each antenna was located at $8 \mathrm{~cm}$ of depth aligned vis-à-vis in co-polarized mode. An antenna was fixed at a distance of $4 \mathrm{~cm}$ from the phantom's internal edge whereas the second one was moved away from a starting distance $d=20 \mathrm{~mm}$ up to $d=140 \mathrm{~mm}$ in steps of 10 mm (Fig. 4).

The respective discrete scatter plots of $P L\left(d_{i}\right)$ for the frequency bands of $2.36-2.4 \mathrm{GHz}$ and $2.4-2.5 \mathrm{GHz}$ are shown in Fig. 5. As seen, the difference in path loss between these two bands is negligible for all the considered distance points. Therefore, in order to produce a path loss formula the channel measurements were averaged over the entire frequency range of 2.36-2.5 GHz. The resulting scatter plot and fitted curve for 2.36-2.5 GHz are shown in Fig. 6. 


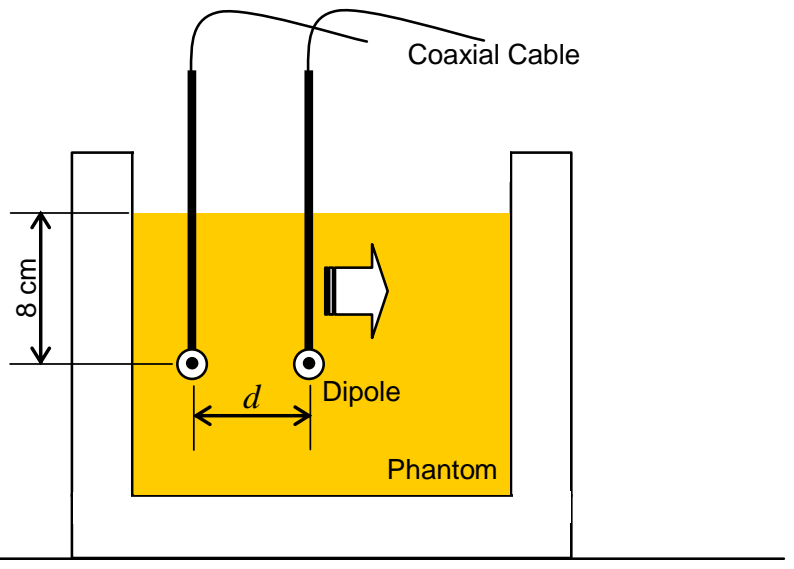

Fig. 4. Measurement setup for the IB2IB channel scenario.

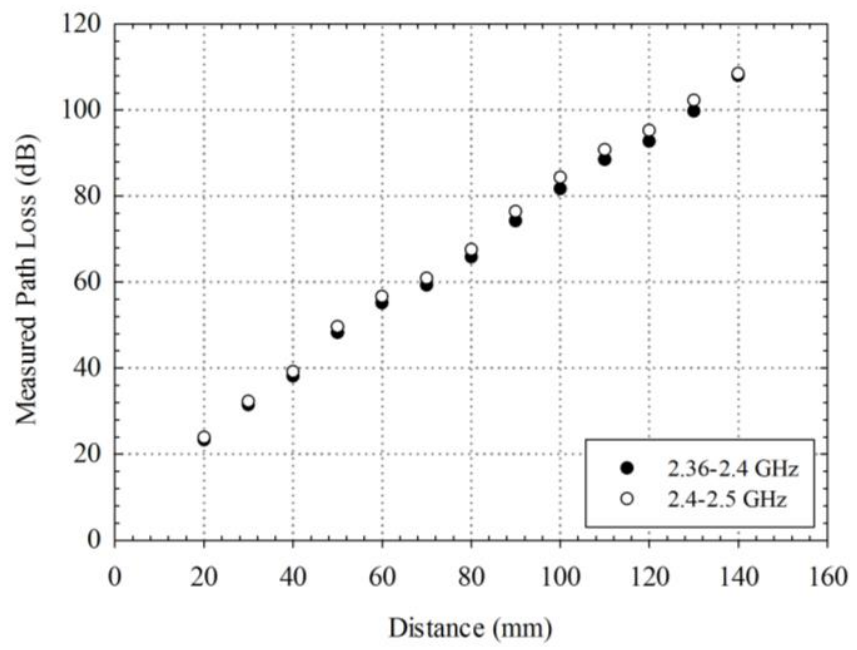

Fig. 5. IB2IB scatter plots of path loss for $2.36-2.4 \mathrm{GHz}$ and $2.4-2.5 \mathrm{GHz}$.

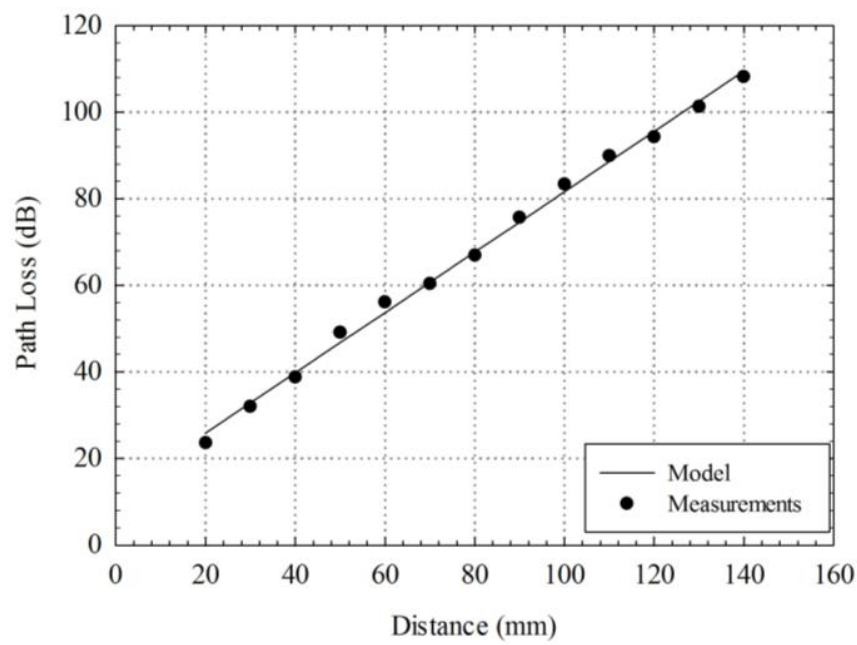

Fig. 6. IB2IB scatter plot and fitted curve of path loss for $2.36-2.5 \mathrm{GHz}$.

In this case, the mathematical model (fitted curve) for the path loss in $\mathrm{dB}$ as a function of $d$ in millimeters is a linear equation given as

$$
P L(d)=P L_{0}+\alpha d,
$$

where $P L_{0}$ is an initial path loss value in $\mathrm{dB}$ observed in the limit when $d \rightarrow 0$, and $\alpha$ is a scaling factor. Here, $P L_{0}=11.9 \mathrm{~dB}$ and $\alpha=0.7 \mathrm{~dB} / \mathrm{mm}$. This model is valid for $20 \leq d \leq 140 \mathrm{~mm}$. For the interval $20 \leq d \leq 80 \mathrm{~mm}$ and similar dielectric properties of the propagation medium, this model is in good agreement with the channel simulation and measurement results reported in [27], [28], where IB2IB path loss formulas valid for $5 \leq d \leq 80 \mathrm{~mm}$ were introduced.

\section{B. In-Body to On-Body (IB2OB) Channel Scenario}

In this scenario one insulated dipole was submerged in the phantom whereas the helical antenna (Fig. 2(b)) was facing it at the same height in direct contact with the external phantom's wall. We denoted the distance from the insulated dipole to the phantom's internal wall as $d_{\text {in }}$. Notice that in this case the effective distance separating the two antennas in millimeters including the phantom's wall thickness, $w_{\text {th }}$, is

$$
d=d_{\text {in }}+w_{\text {th }} .
$$

However, we measured the path loss through the $40 \mathrm{~mm}$ thick Styrofoam ${ }^{\mathrm{TM}}$ wall and found it to be negligible, therefore one can assume that

$$
P L\left(d_{\text {in }}\right) \approx P L(d) .
$$

In a real-life IB2OB scenario where a wearable sensor is in direct contact with a patient's skin, $d_{\mathrm{in}}=d$ and (3) may become $P L\left(d_{\text {in }}\right)=P L(d)$. Thus the helical antenna was kept fixed whereas $d_{\mathrm{in}}$ was varied by moving the dipole away in steps of $10 \mathrm{~mm}$ as shown in Fig. 7. The scatter plot and fitted curve as a function of $d_{\text {in }}$ for $2.36-2.5 \mathrm{GHz}$ are shown in Fig. 8. A linear equation of the same form as (1) is a good model for the path loss in this case too, with $P L_{0}=29.7 \mathrm{~dB}$ and $\alpha=0.6 \mathrm{~dB} / \mathrm{mm}$. This model is valid for $10 \leq d_{\mathrm{in}} \leq 80 \mathrm{~mm}$.

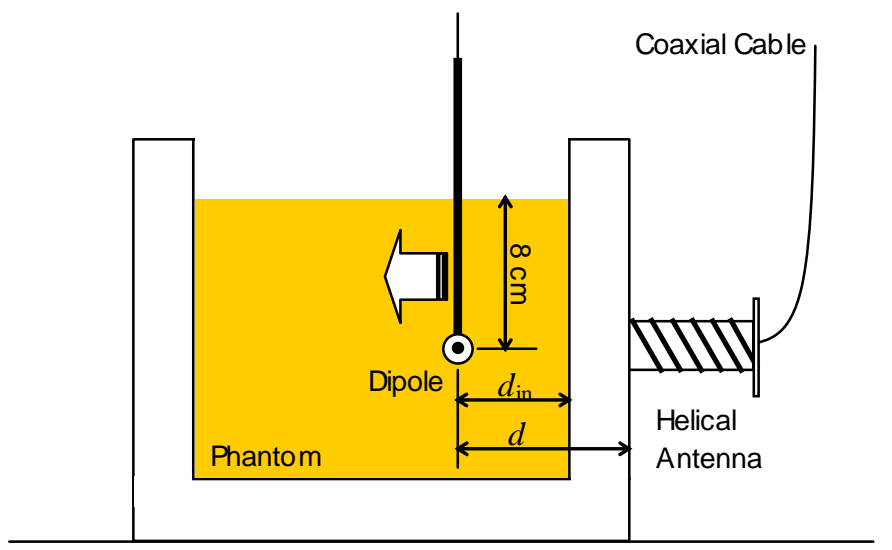

Fig. 7. Measurement setup for the IB2OB channel scenario. 


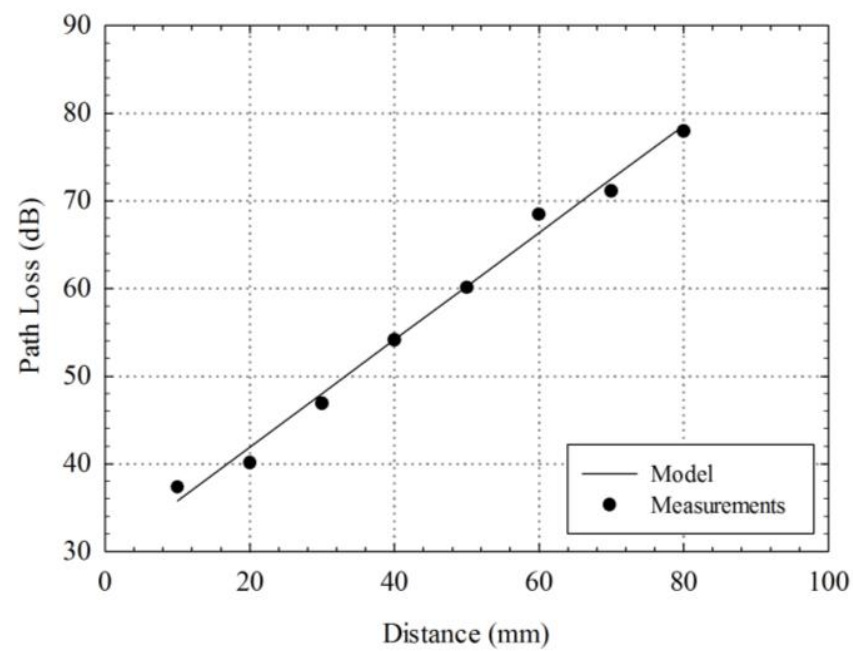

Fig. 8. IB2OB scatter plot and fitted curve of path loss for 2.36-2.5 GHz.

Notice that for the same distance value a larger path loss is observed in the IB2OB channel scenario when compared to IB2IB. This additional loss is a direct result of the impedance mismatch caused by the change of propagation medium along the propagation path [31]-[33].

\section{In-Body to Off-Body (IB2OFF) Channel Scenario}

The measurement setup for the IB2OFF scenario is similar to that of IB2OB, but here the insulated dipole was fixed at a distance $d_{\text {in }}$ as in the previous scenario whereas the helical antenna was moved away from the phantom. We denoted the distance from the phantom's internal wall to the helical antenna as $d_{\text {off }}$ (Fig. 9). We considered three different fixed values for $d_{\text {in }}: 10 \mathrm{~mm}, 20 \mathrm{~mm}$, and $30 \mathrm{~mm}$. For each of these cases we put the helical antenna at multiple locations so that $d_{\text {off }}$ varied from $40 \mathrm{~mm}$ to $540 \mathrm{~mm}$. The resulting scatter plots and fitted curves as functions of $d_{\text {off }}$ for $2.36-2.5 \mathrm{GHz}$ are presented in Fig. 10.

In this scenario, the fitted curve for the path loss in each case follows a log-distance formula expressed in $\mathrm{dB}$ as

$$
P L_{d_{\text {in }}}\left(d_{\text {off }}\right)=P L_{0_{d_{\text {in }}}}+10 \gamma_{d_{\text {in }}} \log _{10}\left(\frac{d_{\text {off }}}{d_{\text {ref }}}\right) .
$$

where $\gamma$ is a path loss exponent and $d_{\text {ref }}$ is a reference distance equal to $1 \mathrm{~mm}$. The subscript $d_{\text {in }}$ in (4) indicates that the formula is valid for one of the three specific values of $d_{\text {in }}$ considered herein; the corresponding values of $P L_{0}$ and $\gamma$ for these three cases are given in Table I.

TABLE I

PATH LOSS PARAMETERS FOR IB2OFF CHANNEL SCENARIO

\begin{tabular}{|c|c|c|c|}
\hline & $d_{\text {in }}=10 \mathrm{~mm}$ & $d_{\text {in }}=20 \mathrm{~mm}$ & $d_{\text {in }}=30 \mathrm{~mm}$ \\
\hline$P L_{0}(\mathrm{~dB})$ & 22.5 & 26.3 & 32.4 \\
\hline$\gamma$ & 0.9 & 0.9 & 0.9 \\
\hline
\end{tabular}

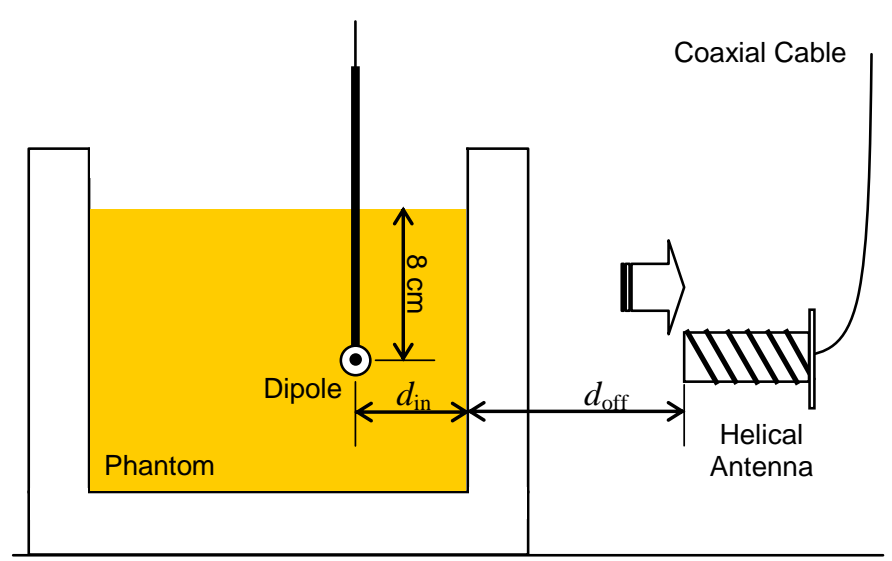

Fig. 9. Measurement setup for the IB2OFF channel scenario.

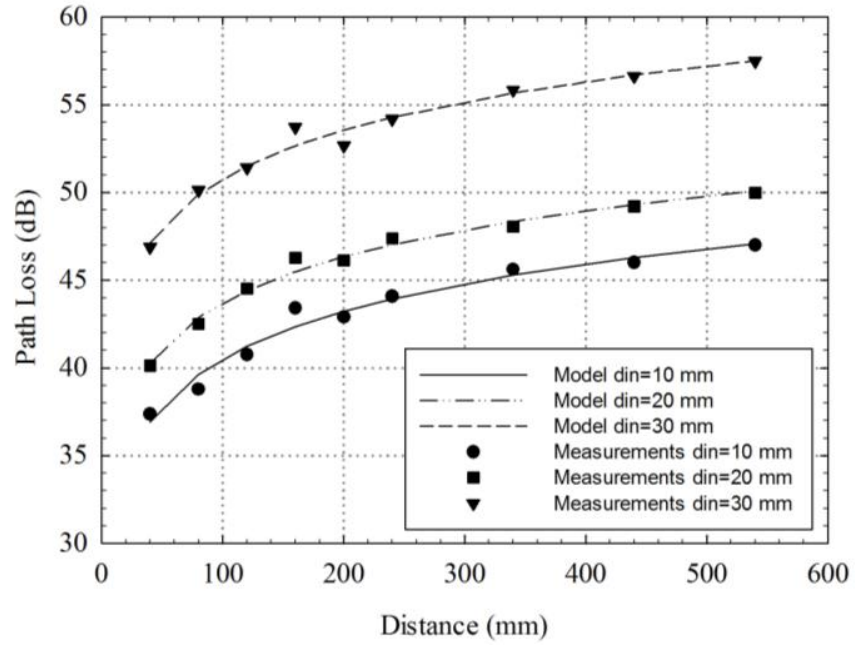

Fig. 10. IB2OFF scatter plots and fitted curves of path loss for $2.36-2.5 \mathrm{GHz}$.

As expected, in this scenario the wave propagation through the aqueous phantom solution was the largest contributor to the total path loss along $d$. This can be observed by comparing Figs. 8 and 10, where it is evident that the rate at which the path loss increases with distance is significantly lower in the air. As hinted in [30], the path loss in the IB2OFF channel scenario can be approximated by considering a combination of IB2OB and indoor path loss models; when no surrounding objects and obstacles are present, then simply the free-space loss can be added to the IB2OB path loss.

\section{DISCUSSION}

The set of path loss formulas presented above are aimed to provide some insight into the behavior of the different in-body channel scenarios to assist the biomedical engineer in the early stage of the design of wireless implantable sensors. It is clear that these path loss models are antenna-dependent as the vast majority of other BAN propagation models proposed in the literature. As the wavelength of the propagating signal in the phantom aqueous solution is $\lambda=17 \mathrm{~mm}$, some of the measurements were inevitably performed within the reactive near-field of each dipole antenna, the boundary of which is 
$R=36 \mathrm{~mm}$. However, implantable and free-space antennas with $\left|S_{11}\right|<-10 \mathrm{~dB}$ were used in all the channel scenarios to try to counter as much as possible the antenna effects on the path loss. Although channel measurements in a homogeneous propagation medium like an aqueous phantom solution do not fully capture the effects of propagation through inhomogeneous multilayer structures like the human body, they provide practical rules of thumb to assess the feasibility of establishing reliable communications for some implantable biomedical applications within 2.36-2.5 GHz.

For instance, consider the ultralow power radio system for BAN applications designed in [34], which can operate in 2.36$2.4 \mathrm{GHz}$ and the $2.4 \mathrm{GHz}$ ISM band. The transmitter (Tx) of this system utilizes a basic on-off keying (OOK) modulation scheme with $0 \mathrm{dBm}$ peak power. Including the Tx baseband, the Tx power consumption is $4.243 \mathrm{~mW}$ when transmitting a logical "1" and $0.919 \mathrm{~mW}$ for a logical "0." The receiver (Rx) front end achieves a sensitivity of $-75 \mathrm{dBm}$ at $5 \mathrm{Mbps}$ and $-78 \mathrm{dBm}$ at $3 \mathrm{Mbps}$. Therefore, if this radio system is equipped with properly matched implantable/on-body antennas accordingly, the maximal transmission range that may be attained for the different in-body scenarios can be estimated with the path loss formulas we provided.

From (1) it follows that for IB2IB the maximal transmission range of this BAN system is $90 \mathrm{~mm}$ and $94 \mathrm{~mm}$ for $5 \mathrm{Mbps}$ and $3 \mathrm{Mbps}$, respectively. These transmission ranges are suitable for some communication applications in the thoracic cavity, e.g., wireless sensing for a subcutaneous implantable cardioverter-defibrillator (s-ICD) [35], [36]. An implanted cardiovascular pressure monitor integrated with a medical stent [37] could send an alert signal on a wireless interface within $2360-2483.5 \mathrm{MHz}$ to the s-ICD in order to deliver transthoracic shocks when ventricular tachyarrhythmias are detected.

Similarly, for IB2OB the maximal transmission range is 75 $\mathrm{mm}$ and $80 \mathrm{~mm}$ for $5 \mathrm{Mbps}$ and $3 \mathrm{Mbps}$, respectively. These transmission ranges represent the maximal implantation depths from the skin for the wireless sensors. Hence, applications like the insertable loop recorder (ILR) [38] and continuous glucose monitoring (CGM) systems [39] could also benefit from smaller antenna sizes by using radio interfaces within 2360-2483.5 MHz. Since these applications utilize a subcutaneous wireless sensor, i.e., a sensor implanted just under the patient's skin, the transmission range could likely be extended to IB2OFF for communication with a handheld patient assistant unit to display, analyze, and record the physiological data. On the other hand, the above transmission ranges for IB2OB within $2360-2483.5 \mathrm{MHz}$ may not fulfill the transmission range requirements for some electronic pill applications like the wireless capsule endoscope (WCE) [11]. In this biomedical application the transmission range varies widely as the WCE travels inside the gastrointestinal (GI) tract transmitting images to a belt receiver/recorder that the patient has to wear during the entire endoscopic procedure (approximately 8 hours). An average transmission range of $200 \mathrm{~mm}$ is typically required for a WCE procedure, but up to
$500 \mathrm{~mm}$ may be needed in some cases. Therefore, practical solutions like the use of a matching layer and spatial diversity reception that have been studied in the context of ultra wideband (UWB) WCE [40]-[42] should be also investigated for 2360-2483.5 MHz.

Nevertheless, the designer of an implantable communication link using our path loss models has to take into account the fact that miniaturized implantable antennas may not be so well matched to the medium as the dipoles used in our measurements. Implantable antenna mismatch and other varying factors like the different thicknesses of fat layer of the patients require the addition of a link budget margin to the path loss calculation. The value of such margin is generally determined based on practical prototype tests.

\section{CONCLUSIONS}

We have presented a collection of path loss models for 2.36-2.5 GHz derived from measurements in a liquid phantom that reproduced the dielectric characteristics of human muscle tissues. These results provide useful insight into the behavior of the different radio channel scenarios for implant communications. However, the fact that the measurements were performed using a homogeneous propagation medium leaves some room for improvement. Therefore, our future work considers the performance of in-vivo channel measurements in an animal subject. Such a measurement campaign will allow capturing the effects of blood circulation, respiration, and temperature gradients on the path loss for the different in-body channel scenarios, which are not properly modeled in most cases; even numerical simulations using digital anatomical models often fail to capture these effects. From the experience gained after multiple in-vivo tests of implantable radio transceivers at the Intervention Centre, Oslo University Hospital [43], [44], we have concluded that channel measurements on a living porcine subject can produce path loss formulas that fairly approximate the propagation characteristics in the human torso. It will be necessary, however, to perform the measurements using various types of antennas with significantly variable sizes in order to fully validate the parameters of the formulas presented in this paper.

Our current path loss models for the different in-body channel scenarios indicate that radio interfaces within 2.36-2.5 $\mathrm{GHz}$ are feasible for some implantable biomedical sensors. The use of these frequency bands can promote further miniaturization of the antennas, a very important design aspect for biomedical implants. At the same time, wider channel bandwidths will be available for the transmission of larger amounts of physiological data. The design of radio transceivers for implants operating within $2.36-2.5 \mathrm{GHz}$ will be a part of our future research activities too. Optimal transceiver design, however, calls for more accurate characterization of the different in-body radio channel scenarios. Hence, this study could contribute to spark further research interest toward the exploitation of this portion of the spectrum for implant communications. 


\section{REFERENCES}

[1] R. Chávez-Santiago and I. Balasingham, "Ultrawideband signals in medicine [Life Sciences]," IEEE Signal Process. Mag., vol. 31, no. 6, pp. 130-136, Nov. 2014.

[2] H. Alemdar and C. Ersoy, "Wireless sensor networks for healthcare: A survey," Computer Networks, vol. 54, no. 15, pp. 2688-2710, Oct. 2010.

[3] E. Jovanov and A. Milenkovic, "Body area networks for ubiquitous healthcare applications: Opportunities and challenges," J. Med. Syst., vol. 35, no. 5, pp. 1245-1254, Oct. 2011.

[4] G. D. Clifford and D. Clifton, "Wireless sensor technology in disease management and medicine," Annual Review of Medicine, vol. 63, pp. 479-492, Feb. 2012.

[5] IEEE Standard for Local and Metropolitan Area Networks-Part 15.6: Wireless Body Area Networks, IEEE Standard 802.15.6-2012, 2012.

[6] R. de Francisco and A. Pandharipande, "Spectrum occupancy in the 2.36-2.4 GHz band: Measurements and analysis," in Proc. European Wireless Conf. (EW), Lucca, Italy, 2010, pp. 231-237.

[7] T. Falck and D. Wang, "Biosensor communication technology and standards," in Handbook of Biomedical Telemetry, K. S. Nikita, Ed. Hoboken, NJ: John Wiley \& Sons, 2014, pp. 330-367.

[8] A Statistical Path Loss Model for MICS, IEEE P802.15-08-0519-010006, September 8, 2008.

[9] K. Sayrafian-Pour, W.-B. Yang, J. Hagedorn, J. Terrill, K. Y. Yazdandoost and K. Hamaguchi, "Channel models for medical implant communication," International Journal of Wireless Information Networks, vol. 17, no. 3-4, pp. 105-112, December 2010.

[10] R. Chávez-Santiago, K. Sayrafian-Pour, A. Khaleghi, K. Takizawa, J. Wang, I. Balasingham and H.-B. Li, "Propagation models for IEEE 802.15.6 standardization of implant communication in body area networks," IEEE Commun. Mag., vol. 51, no. 8, pp. 80-87, Aug. 2013.

[11] M. R. Yuce and T. Dissanayake, "Easy-to-swallow wireless telemetry," IEEE Microwave, vol. 13, no. 6, pp. 90-101, Sep./Oct. 2012.

[12] E. Y. Chow, M. M. Morris and P. Irazoqui, "Implantable RF medical devices," IEEE Microwave, vol. 14, no. 4, pp. 64-73, Jun. 2013.

[13] R. Chávez-Santiago and I. Balasingham, "Radio propagation models for in-body sensors," in Proc. XXXIth URSI General Assembly and Scientific Symp. (URSI GASS), Beijing, China, 2014, pp. 1-4.

[14] T. Karacolak, A. Z. Hood and E. Topsakal, "Design of a dual-band implantable antenna and development of skin mimicking gels for continuous glucose monitoring," IEEE Trans. Microwave Theory Tech., vol. 56, no. 4, pp. 1001-1008, Apr. 2008.

[15] W. Xia, K. Saito, M. Takahashi and K. Ito, "Performances of an implanted cavity slot antenna embedded in the human arm," IEEE Trans. Antennas Propagat., vol. 57, no. 4, pp. 894-899, Apr. 2009.

[16] E. Y. Chow, Y. Ouyang, B. Beier, W. J. Chappell and P. P. Irazoqui, "Evaluation of cardiovascular stents as antennas for implantable wireless applications," IEEE Trans. Microwave Theory Tech., vol. 57, no. 10, pp. 2523-2532, Oct. 2009.

[17] M. L. Scarpello, D. Kurup, H. Rogier, D. V. Ginste, F. Axisa, J. Vanfleteren, W. Joseph, L. Martens and G. Vermeeren, "Design of an implantable slot dipole conformal flexible antenna for biomedical applications," IEEE Trans. Antennas Propagat., vol. 59, no. 10, pp. 3556-3564, Oct. 2011.

[18] C.-K. Wu, T.-F. Chien, C.-L.Yang and C.-H. Luo, "Design of novel Sshaped quad-band antenna for MedRadio/WMTS/ISM implantable biotelemetry applications," International Journal of Antennas and Propagation, vol. 2012, pp. 1-12, 2012.

[19] S. A. Kumar and T. Shanmuganantham, "Implanted CPW fed monopole antenna for biomedical applications," Advances in Computing and Information Technology, vol. 178, pp. 97-105, 2013.

[20] M. R. Yuce and T. Dissanayake, "Easy-to-swallow antenna and propagation," IEEE Microwave, vol. 14, no. 4, pp. 74-82, Jun. 2013.

[21] S. A. Kumar and T. Shanmuganantham, "Design and analysis of implantable CPW fed bowtie antenna for ISM band applications," $A E U$ International Journal of Electronics and Communications, vol. 68, no. 2, pp. 158-165, Feb. 2014.

[22] S. A. Kumar and T. Shanmuganantham, "Coplanar waveguide-fed ISM band implantable crossed-type triangular slot antenna for biomedical applications," International Journal of Microwave and Wireless Technologies, vol. 6, no. 2, pp. 167-162, Apr. 2014.

[23] Z. Duan, Y.-X. Guo, M. Je and D.-L. Kwong, "Design and in vitro test of a differentially fed dual-band implantable antenna operating at MICS and ISM bands," IEEE Trans. Antennas Propagat., vol. 62, no. 5, pp. 2430-2439, May 2014
[24] S. A. Kumar and T. Shanmuganantham, "Design of implantable CPW fed monopole H-slot antenna for $2.45 \mathrm{GHz}$ ISM band applications," AEU-International Journal of Electronics and Communications, vol. 68, no. 7, pp. 661-666, Jul. 2014.

[25] J. Gemio, J. Parrón and J. Soler, "Human body effects on implantable antennas for ISM bands applications: Models comparison and propagation losses study," Progress in Electromagnetics Research, vol. 110, pp. 437-452, 2010.

[26] A. Alomainy and Y. Hao, "Modeling and characterization of biotelemetric radio channel from ingested implants considering organ contents," IEEE Trans. Antennas and Propag., vol. 57, no. 4, pp. 9991005, Apr. 2009.

[27] D. Kurup, W. Joseph, G. Vermeeren and L. Martens, "Path loss model for in-body communication in homogeneous human muscle tissue," Electronic Letters, vol. 45, no. 9, pp. 453-454, Apr. 2009.

[28] D. Kurup, W. Joseph, G. Vermeeren and L. Martens, "In-Body path loss model for homogeneous human tissues," IEEE Trans. Electromagn. Compat., vol. 54, no. 3, pp. 556-564, Jun. 2012.

[29] C. K. Chou, G. W. Chen, A. W. Guy and K. H. Luk, "Formulas for preparing phantom muscle tissue at various radiofrequencies," Bioelectromagnetics, vol. 5, no. 4, pp. 435-441, Oct. 1984.

[30] Channel Model for Body Area Network (BAN), IEEE P802.15-08-078012-0006, November 10, 2010.

[31] T. Dissanayake, K. P. Esselle and M. R. Yuce, "Dielectric loaded impedance matching for wideband implanted antennas," IEEE Trans. Microwave Theory Tech., vol. 57, no. 10, pp. 2480-2487, Oct. 2009.

[32] A. Khaleghi, R. Chávez-Santiago and I. Balasingham, "On the use of a dielectric matching layer for ultra wideband medical applications," in Proc. 7th Int. Conf. on Body Area Networks (BodyNets), Oslo, Norway, 2012, pp. 69-75.

[33] R. Chávez-Santiago, A. Khaleghi and I. Balasingham, "Matching layer for path loss reduction in ultra wideband implant communications," in Proc. 36th Annual Int. Conf. of the IEEE Engineering in Medicine and Biology Society (EMBC), Chicago, IL, 2014, pp. 6989-6992.

[34] M. Vidojkovic, L. Huang, J. Penders, G. Dolmans and H. de Groot, "Ultralow power radio design for emerging healthcare applications," in Low Power Emerging Wireless Technologies, R. Mahmoudi and K. Iniewski, Ed. Boca Raton, FL: CRC Press, 2013, pp. 253-272.

[35] G. H. Bardy et al., "An entirely subcutaneous implantable cardioverterdefibrillator," N. Engl. J. Med., vol. 363, no. 1, pp. 36-44, Jul. 2010.

[36] R. G. Hauser, "The subcutaneous implantable cardioverter-defibrillator: Should patients want one?," J. Am. Coll. Cardiol., vol. 61, no. 1, pp. 2022, Jan. 2013.

[37] E. Y. Chow, A. L. Chlebowski, S. Chakraborty, W. J. Chappel and P. P. Irazoqui, "Fully wireless implantable cardiovascular pressure monitor integrated with a medical stent," IEEE Trans. Biomed. Eng., vol. 57, no. 6, pp. 1487-1496, Jun. 2010.

[38] A. D. Krahn, G. J. Klein, A. C. Skanes and R. Yee, "Insertable loop recorder use for detection of intermittent arrhythmias," Pacing and Clinical Electrophysiology, vol. 27, no. 5, pp. 657-664, May 2004.

[39] R. Hovorka, "Continuous glucose monitoring and closed-loop systems," Diabetic Medicine, vol. 23, no. 1, pp. 1-12, Jan. 2006.

[40] J. Shi, D. Anzai and J. Wang, "Channel modeling and performance analysis of diversity reception for implant UWB wireless link," IEICE Trans. Commun., vol. E95-B, no. 10, pp. 3197-3205, Oct. 2012.

[41] R. Chávez-Santiago, J. Wang and I. Balasingham, "The ultra wideband capsule endoscope," IEEE Int. Conf. on Ultra-Wideband (ICUWB), Sydney, Australia, 2013, pp. 72-78.

[42] K. Thotahewa, J.-M. Redoute and M. R. Yuce, "A UWB wireless capsule endoscopy device," in Proc. $36^{\text {th }}$ Annual Int. Conf. of the IEEE Engineering in Medicine \& Biology Society (EMBC), Chicago, IL, 2014, pp. 6977-6980.

[43] R. Chávez-Santiago, I. Balasingham, J. Bergsland, W. Zahid, K. Takizawa, R. Miura and H.-B. Li, "Experimental implant communication of high data rate video using an ultra wideband radio link," in Proc. 35th Annual Intl. Conf. of the IEEE Engineering in Medicine \& Biology Society (EMBC), Osaka, Japan, 2013, pp. 51755178.

[44] A. Daisuke, K. Katsu, R. Chávez-Santiago, Q. Wang, D. Plettemeier, J. Wang and I. Balasingham, "Experimental evaluation of implant UWBIR transmission with living animal for body area networks," IEEE Trans. Microw. Theory Techn., vol. 62, no. 1, pp. 183-192, Jan. 2014. 